Research Article

\title{
On the Global Asymptotic Stability of Solutions of Some Difference Equations with Intrinsic Initial Conditions
}

\author{
Vasile Berinde \\ Department of Mathematics and Computer Science, Technical University of Cluj-Napoca, North University Center at Baia Mare, \\ Victoriei 76, Baia Mare 430122, Romania \\ Correspondence should be addressed to Vasile Berinde; vberinde@cunbm.utcluj.ro
}

Received 2 December 2020; Revised 26 December 2020; Accepted 21 January 2021; Published 31 January 2021

Academic Editor: Praveen Agarwal

Copyright (c) 2021 Vasile Berinde. This is an open access article distributed under the Creative Commons Attribution License, which permits unrestricted use, distribution, and reproduction in any medium, provided the original work is properly cited.

Our aim in this paper is to study the asymptotic global stability of the positive solutions for a class of first-order nonlinear difference equations with a remarkable feature: the initial conditions are intrinsic and not explicitly given. Global stability results are obtained in a particular case and then for a general class of first-order difference equations. We also provide the results of some numerical experiments obtained by the mini software package FIXPOINT to illustrate asymptotic global stability as well as the rate of convergence. To the best of our knowledge, our approach is the first one in the literature on the stability of difference equations without explicit initial conditions and might generate an interesting new direction of further studies.

\section{Introduction}

Many important phenomena and dynamical processes in several fields of applied sciences and engineering are described by means of differential and/or difference equations. On the contrary, difference equations also appear naturally in connection with discretization schemes applied to ordinary or partial differential equations. For such a difference equation or system of difference equations, it is important to study the local and global behaviour of their solutions. This behaviour is influenced in many instances by the initial conditions associated to the given difference equations, making it an important issue in the study of concrete dynamical processes.

It is the main aim of this paper to study the asymptotic global stability of the positive solutions for a general class of first-order nonlinear difference equations with a remarkable feature: the initial conditions are not explicitly given but are intrinsic.

Our approach is motivated mainly by the fact that, in the case of nonlinear difference equations, properties of solutions, in most of the concrete situations, can only be observed and conjectured by numerical simulations, and it is extremely difficult to obtain them analytically. Therefore, it is fundamentally important to identify those classes of nonlinear difference equations for which we still can perform analytically a qualitative study of the properties of their solutions, particularly the global behaviour, which is the topic of the current study.

To the best of our knowledge, our approach is the first one in the literature on the stability of difference equations with no explicit initial conditions and might generate an interesting new direction of further studies.

\section{Preliminaries}

In most of the studies devoted to the global stability of $k$-order difference equations, the appropriate initial conditions are explicitly assumed and the proofs are essentially based on these conditions. For example, in [1], the authors studied the dynamics and the global asymptotic stability of the second-order difference equation:

$$
y_{n+1}=A+\frac{y_{n}}{y_{n-1}}, \quad n=0,1, \ldots
$$

with the initial conditions $y_{-1}, y_{0} \in(0, \infty)$, where $A>0$.

It was shown in [1] that the unique positive equilibrium $\bar{y}=1+A$ of equation (1) is globally asymptotically stable. 
In [2], some partial answers to Conjecture 6.4.1 and Open problem 6.4.1 in [1] were given by obtaining a sufficient condition for the global asymptotic stability of the unique positive equilibrium of the more general $(k+1)$-order difference equation:

$$
y_{n+1}=A+\frac{y_{n}}{y_{n-k}}, \quad n=0,1, \ldots
$$

with the initial conditions $y_{-k}, \ldots, y_{0} \in(0, \infty)$, where $A>0$ and $k \in\{2,3, \ldots\}$. More recently, El-Owaidy et al. [3], Stević [4-7], and many other authors have studied the dynamics of the difference equations in the family:

$$
x_{n+1}=\alpha+\frac{x_{n}^{p}}{x_{n}^{p}}, \quad n=0,1, \ldots,
$$

with the initial condition $x_{0}>0$, where $\alpha \in[0, \infty)$ and $p \in[1, \infty)$. In continuation of this research work, Aloqeili [8] studied the asymptotic behaviour of the rational difference equation:

$$
x_{n+1}=\alpha+\frac{x_{n}^{p}}{x_{n-1}^{p}}, \quad n=0,1, \ldots,
$$

with the initial conditions $x_{-1}, x_{0} \in(0,+\infty)$, where $\alpha \in[0, \infty)$ and $p \in(0,1)$.

Aloqeili [8] also studied a more general difference equation, i.e.,

$$
x_{n+1}=\alpha+\frac{x_{n}^{p}}{x_{n-k}^{p}}, \quad n=0,1, \ldots,
$$

with the initial conditions $x_{-k}, \ldots, x_{0} \in(0,+\infty)$, where $\alpha \in[0, \infty), p \in(0,1)$, and $k \in\{1,2, \ldots\}$.

We note that the technique of proof in [1-24] and in many other related papers is essentially based on the linearized stability theorem, on the one hand, and on the initial conditions, which are in almost all cases restricted to positive values, on the other hand.

Starting from these facts, as announced in Section 1, our aim in this paper is to obtain asymptotic stability results for a class of difference equations with intrinsic initial conditions.

To this end, we need some basic notions and results used in the study of difference equations. We shall be mainly concerned with the particular case $X:=I$, where $I \subset \mathbb{R}$ is an interval. In this context, a fixed point $x^{*}$ of $f$ is usually called an equilibrium point of the first-order difference equation $x_{n+1}=f\left(x_{n}\right), n \geq 0$.

Reminding, see for example [18], that the equilibrium point $x^{*}$ of the $k$-order difference equation

$$
x_{n+1}=T\left(x_{n}, \ldots, x_{n-k+1}\right), \quad n=k-1, k, k+1, \ldots,
$$

is said to be locally stable if, for every $\epsilon>0$, there exists $\delta>0$ such that, for all $x_{0}, x_{1}, \ldots, x_{k-1} \in I$ satisfying

$$
\left|x_{0}-x^{*}\right|+\left|x_{1}-x^{*}\right|+\cdots+\left|x_{k-1}-x^{*}\right|<\delta \text {, }
$$

one has

$$
\left|x_{n}-x^{*}\right|<\epsilon, \quad \forall n \geq 0 .
$$

The equilibrium point $x^{*}$ of (6) is said to be stable if $x^{*}$ is a locally stable solution and there exists $\delta>0$ such that, for all $x_{0}, x_{1}, \ldots, x_{k-1} \in I$ satisfying (7), one has

$$
\lim _{n \rightarrow \infty} x_{n}=x^{*} \text {. }
$$

The equilibrium point $x^{*}$ of (6) is a global attractor if, for all $x_{0}, x_{1}, \ldots, x_{k-1} \in I$, one has

$$
\lim _{n \rightarrow \infty} x_{n}=x^{*} \text {. }
$$

The equilibrium point $x^{*}$ of (6) is globally asymptotically stable if $x^{*}$ is simultaneously locally stable and a global attractor of (6).

The equilibrium point $x^{*}$ of (6) is unstable if it is not locally stable.

\section{Global Asymptotic Stability of First-Order Difference Equations with Intrinsic Initial Conditions}

We start by studying the stability of the solutions of a simple first-order difference equation with intrinsic initial condition.

Theorem 1. The equilibrium point $x^{*}=3$ of the difference equation

$$
x_{n+1} x_{n}-2 x_{n}=3, \quad n=0,1,2, \ldots,
$$

is globally asymptotically stable.

Proof. First of all, we note that no explicit initial condition is associated with the difference equation (11). It is also easy to see that the equilibriums of equation (11) are the roots of the quadratic equation $x^{2}-2 x-3=0$, that is, $x^{*} \in\{-1,3\}$.

We shall prove now that the equilibrium point $x^{*}=3$ is globally asymptotically stable. Assume there exists $m \in \mathbb{N}^{*}$ such that $x_{m}=3$. Then, by (11), $x_{m-1}=3, \ldots, x_{1}=3$ and $x_{0}=3$. Moreover, one can show by induction that, in this case, we also have $x_{n}=3$, for all $n>m$.

Therefore, if one single term of the sequence $\left\{x_{n}\right\}$ would be equal to 3 , in particular, if $x_{0}=3$, then all its terms have the same value and so the equilibrium $x^{*}=3$ is globally asymptotically stable.

Furthermore, let us assume that $x_{n} \neq 3$, for all $n \geq 0$. In this case, we can denote

$$
y_{n}=\frac{1}{x_{n}-3}, \quad n \geq 0 .
$$

Note also that $x_{n} \neq 2$, for all $n \geq 0$. Indeed, if we would have $x_{m}=2$ for a certain $m \in \mathbb{N}$, then, by (11), we would get $0=3$, a contradiction.

So, by (11), we have 


$$
\begin{aligned}
y_{n} & =\frac{1}{x_{n}-3}=\frac{1}{\left(3 /\left(x_{n+1}-2\right)\right)-3}=\frac{x_{n+1}-2}{3\left(3-x_{n+1}\right)} \\
& =-\frac{1}{3}-\frac{1}{3} \cdot \frac{1}{x_{n+1}-3},
\end{aligned}
$$

which shows that the sequence $\left\{y_{n}\right\}$ satisfies the linear recurrence relation

$$
y_{n+1}=-3 y_{n}-1, \quad n \geq 1,
$$

which can be written in a telescopic form as

$$
\frac{y_{n+1}}{(-3)^{n+1}}=\frac{y_{n}}{(-3)^{n}}-\frac{1}{(-3)^{n+1}}, \quad n \geq 1 .
$$

Now, by summing up the $n$ relations obtained from (15) by letting $n:=p, p+1, \ldots, n+p-1$,

$$
\begin{gathered}
\frac{y_{p+1}}{(-3)^{p+1}}=\frac{y_{p}}{(-3)^{p}}-\frac{1}{(-3)^{p+1}}, \\
\frac{y_{p+2}}{(-3)^{p+2}}=\frac{y_{p+1}}{(-3)^{p+1}}-\frac{1}{(-3)^{p+2}}, \\
\quad \ldots \\
\frac{y_{n+p}}{(-3)^{n+p}}=\frac{y_{n+p-1}}{(-3)^{n+p-1}}-\frac{1}{(-3)^{n+p}},
\end{gathered}
$$

one obtains

$$
\frac{y_{n+p}}{(-3)^{n+p}}=\frac{y_{p}}{(-3)^{p}}-\sum_{k=p}^{n+p-1} \frac{1}{(-3)^{k+1}},
$$

which yields, after computing the geometric progression sum,

$$
y_{n+p}=(-3)^{n} \cdot y_{p}+\frac{(-3)^{n}-1}{4}, \quad n, p \geq 0 .
$$

Now, in view of (12), it follows that $\left\{x_{n}\right\}$ satisfies

$$
x_{n+p}=\frac{x_{p}-3+3\left(x_{p}+1\right)(-3)^{n}}{3-x_{p}+\left(x_{p}+1\right)(-3)^{n}}, \quad n, p \geq 0 .
$$

Since all transformations that lead us to (19) were well determined, we conclude that the denominator of the fraction in (19) is always nonzero, that is,

$$
x_{p} \neq \frac{(-3)^{n}+3}{1-(-3)^{n}}, \quad n=1,2, \ldots ; p=0,1,2, \ldots
$$

Hence, if we denote by $E$ the set of excluded values for any term of the sequence $\left\{x_{n}\right\}$, i.e.,

$$
E=\left\{\frac{(-3)^{n}+3}{1-(-3)^{n}}: \quad n=1,2, \ldots\right\},
$$

then we conclude that the intrinsic initial condition for the difference equation (11) is

$$
x_{0} \in \mathbb{R} / E \text {. }
$$

Now, under the intrinsic initial condition (22), by simply letting $n \longrightarrow \infty$ in (19), we obtain

$$
\lim _{n \longrightarrow \infty} x_{n}=3
$$

which shows that the equilibrium point $x^{*}=3$ of (11) is globally asymptotically stable.

Remark 1.

(a) Theorem 1 above extends Theorem 3.1 in [25], see also [26], where the explicit initial condition was $x_{0} \in(0,+\infty)$, while in Theorem 1 , it is intrinsic.

(b) We note that the points $0,-(3 / 2)$, and $-(6 / 7)$ which are the borders of the set of initial conditions in Theorem 3.3 in [26] are actually the first three elements $e_{n}$ of the set $E$ defined above and are obtained for $n=1, n=2$, and $n=3$, respectively.

(c) It is easy to check that

$$
e_{n}=\frac{(-3)^{n}+3}{1-(-3)^{n}} \longrightarrow-1, \quad \text { as } n \longrightarrow \infty,
$$

which explains why the equilibrium point $y^{*}=-1$ of the difference equation (11) is unstable.

One may prove similarly the following more general result.

Theorem 2. Let $a, b \in \mathbb{R}^{*}, a \neq b$, and $|a|>|b|$. Then, the equilibrium point $x^{*}=a$ of the difference equation

$$
x_{n+1} x_{n}-(a+b) x_{n}+a b=0, \quad n=0,1, \ldots,
$$

is globally asymptotically stable.

Proof. We note that no explicit initial condition is associated with the difference equation (25). It is also easy to see that the equilibriums of the difference equation (25) are the roots of the characteristic equation $x^{2}-(a+b) x+a b=0$, that is, $x^{*} \in\{a, b\}$.

We shall prove now that the equilibrium point $x^{*}=a$ is globally asymptotically stable, while $x^{*}=b$ is unstable. Assume there exists $m \in \mathbb{N}^{*}$ such that $x_{m}=a$. Then, by (25), $x_{m-1}=a, \ldots, x_{1}=a$, and so $x_{0}=a$. Moreover, one can show by induction that, in this case, we also have $x_{n}=a$, for all $n>m$.

Therefore, if one single term of the sequence $\left\{x_{n}\right\}$ would be equal to $a$, in particular, if $x_{0}=a$, then all its terms would have the same value and so the equilibrium $x^{*}=a$ is globally asymptotically stable.

Now, let us assume that $x_{n} \neq a$, for all $n \geq 0$, and denote

$$
y_{n}=\frac{1}{x_{n}-a}, \quad n \geq 0 .
$$

In this case, we also have $x_{n} \neq a+b$, for all $n \geq 0$. Indeed, if we would have $x_{m}=a+b$, for a certain $m \in \mathbb{N}$, then, by (25), we would get immediately $0=a b$, a contradiction. 
So, by (25), we have

$$
y_{n}=\frac{1}{x_{n}-a}=\frac{1}{\left(a b /\left(a+b-x_{n+1}\right)\right)-b}=-\frac{1}{b} \cdot \frac{x_{n+1}-b-a}{x_{n+1}-b},
$$

which shows that, in this way, we have linearized the nonlinear difference equation (25), since $\left\{y_{n}\right\}$ satisfies a linear difference equation:

$$
y_{n+1}=-\frac{b}{a} y_{n}+\frac{1}{a}, \quad n \geq 0 .
$$

Similar to the proof of Theorem 1, one obtains

$$
y_{n+p}=\left(\frac{a}{b}\right)^{n} \cdot y_{p}+\frac{1}{b} \sum_{k=0}^{n-1}\left(\frac{a}{b}\right)^{k}, \quad n, p \geq 0 .
$$

Since $a \neq b$, by evaluating the sum in (29), one obtains

$$
y_{n+p}=\left(\frac{a}{b}\right)^{n} \cdot y_{p}+\frac{1-(a / b)^{n}}{b-a}, \quad n, p \geq 0
$$

which, in view of (26), yields

$$
\frac{1}{x_{n+p}-a}=\frac{x_{p}-a+\left(b-x_{p}\right)(a / b)^{n}}{(b-a))\left(x_{p}-a\right)}, \quad n, p \geq 0 .
$$

Since all transformations that lead us to (31) were equivalent, we conclude that the numerator of the fraction in the right-hand side of (31) is always nonzero, that is,

$$
x_{p} \neq \frac{a b^{n}-b a^{n}}{b^{n}-a^{n}}, \quad n=1,2, \ldots ; p=0,1,2, \ldots
$$

Hence, if we denote by $E$, the set of excluded values for any term of the sequence $\left\{x_{n}\right\}$, i.e.,

$$
E=\left\{\frac{a b^{n}-b a^{n}}{b^{n}-a^{n}}: \quad n=1,2, \ldots\right\},
$$

then we conclude that the intrinsic initial condition for the difference equation (25) is

$$
x_{0} \in \mathbb{R} / E .
$$

Now, by (31), we have

$$
x_{n+p}-a=\frac{(b-a)\left(x_{p}-a\right)}{x_{p}-a+\left(b-x_{p}\right)(a / b)^{n}},
$$

and having in view the fact that $|a|>|b|$, by letting $n \longrightarrow \infty$ in the previous equality, we obtain

$$
\lim _{n \longrightarrow \infty} x_{n}=a,
$$

which shows that the equilibrium point $x^{*}=a$ of (25) is globally asymptotically stable.
Remark 2. (a) One can check that

$$
e_{n}=\frac{a b^{n}-b a^{n}}{b^{n}-a^{n}} \longrightarrow b, \quad \text { as } n \longrightarrow \infty,
$$

which shows that the equilibrium point $b$ of the difference equation (25) is unstable.

(b) If $a=3$ and $b=-1$, then the difference equation (25) in Theorem 2 reduces to the difference equation (11) from Theorem 1.

In the particular case $a=2$ and $b=-1$, by Theorem 2 , we obtain a stability result that extends Theorem 3.1 in [25] to the case of an intrinsic initial condition. Note that, in Theorem 3.1 from [25], the initial condition is $x_{0} \in(0, \infty)$.

Corollary 1. The equilibrium point $x^{*}=2$ of the difference equation

$$
x_{n+1}=1+\frac{2}{x_{n}}, \quad n=0,1, \ldots,
$$

is globally asymptotically stable.

We end the paper with a stability result that covers the case $a=b$.

Theorem 3. If $a \in \mathbb{R}^{*}$, then the equilibrium point $x^{*}=a$ of the difference equation

$$
x_{n+1} x_{n}-2 a x_{n}+a^{2}=0, \quad n=0,1, \ldots,
$$

is globally asymptotically stable.

Proof. It is also easy to see that the unique equilibrium of the difference equation (39) is the double root of the characteristic equation $x^{2}-2 a x+a^{2}=0$, that is, $x^{*}=a$. The case $x_{m}=a$ for some $m$ is trivial. Consider $x_{n} \neq a$ for all $n=0,1,2, \ldots$, and similar to the proof of Theorem 2 , we obtain

$$
\frac{1}{x_{n+p}-a}=\frac{n x_{p}-(n-1) a}{a\left(x_{p}-a\right)}
$$

from which we deduce that the intrinsic initial condition associated to (39) is

$$
x_{0} \neq \frac{n-1}{n} \cdot a
$$

and therefore,

$$
x_{n+p}-a=\frac{a\left(x_{p}-a\right)}{n x_{p}-(n-1) a} \longrightarrow 0, \quad \text { as } n \longrightarrow \infty \text {. }
$$


TABLE 1: Numerical experiments for six values satisfying the intrinsic initial condition corresponding to the difference equation (11).

\begin{tabular}{|c|c|c|c|c|c|c|}
\hline$n$ & $x_{n}$ & $x_{n}$ & $x_{n}$ & $x_{n}$ & $x_{n}$ & $x_{n}$ \\
\hline 0 & -1.01 & -1.001 & 10 & 0.01 & -0.76 & -0.751 \\
\hline 1 & -0.9703 & -0.977 & 2.3 & 302 & -1.94737 & -1.99467 \\
\hline 2 & -1.09184 & -1.009 & 3.304348 & 2.009934 & 0.459459 & 0.495995 \\
\hline 3 & -0.74766 & -0.9732 & 2.907895 & 3.492586 & 8.529412 & 8.048452 \\
\hline 4 & -2.0125 & -1.0827 & 3.031674 & 2.858962 & 2.351724 & 2.372742 \\
\hline 5 & 0.509317 & -0.771 & 2.989552 & 3.049332 & 3.27566 & 3.26436 \\
\hline 6 & 7.890244 & -1.8912 & 3.003495 & 2.983822 & 2.9155846 & 2.919016 \\
\hline 7 & 2.380216 & 0.4137 & 2.998836 & 3.005422 & 3.028861 & 3.027743 \\
\hline 8 & 3.26039 & 9.2516 & 3.000388 & 2.998196 & 2.990471 & 2.990837 \\
\hline 9 & 2.920135 & 2.3243 & 2.999871 & 3.000602 & 3.003186 & 3.003064 \\
\hline 10 & 3.02735 & 3.2907 & 3.000043 & 2.999799 & 2.998939 & 2.99898 \\
\hline$N$ & 20 & 18 & 15 & 16 & 17 & 17 \\
\hline
\end{tabular}

\section{Numerical Experiments}

We performed some numerical experiments for the difference equation (11) and for various values of $x_{0}$ satisfying the intrinsic initial condition. The most significant ones are presented in Table 1.

Except for the initial value $x_{0}=10$, the values of $x_{0}$ in the other five cases were chosen in such a way to be close enough to the unstable equilibrium point $y^{*}=-1$, that is, $x_{0} \in\{-1.01 ;-1.001\}$, or close enough to some points in the exception set $E$, that is, $x_{0} \in\{-0.76 ;-0.751\}$ and $x_{0}=0.1$, respectively. The obtained results are presented in Table 1 and illustrate both the global asymptotical stability of the equilibrium point $x^{*}=3$ and the rate of convergence $(N$ denotes the number of iterations needed to reach the solution, with 6 exact digits).

Note that the rate of convergence of the dynamical system $\left\{x_{n}\right\}$ defined by the difference equation (11) is linear, see [27], that is,

$$
\lim _{n \longrightarrow \infty} \frac{\left|x_{n+1}-3\right|}{\left|x_{n}-3\right|^{1}}=\frac{1}{3}
$$

a fact which is illustrated by the numerical results given in Table 1.

\section{Conclusion}

In this paper, we have studied the asymptotic global stability of the positive solutions for a class of first-order difference equations with an innovative feature: the initial conditions are intrinsic and not explicitly given, as usual in the literature. Global stability results have been obtained first in a particular case and then for a general class of firstorder difference equations. We also illustrate the theoretical results by some numerical experiments obtained by the use of the mini software package FIXPOINT. The numerical tests illustrate both the asymptotic global stability of the solutions and the rate of convergence of the iterative process.

To the best of our knowledge, our approach is the first one to study the stability of solutions for second-order difference equations without explicit initial conditions and might generate an interesting new direction of further studies with important applications in the study of phenomena and dynamical processes from several fields of applied sciences and engineering.

The future work is planned to extend this idea by considering first fractional difference equations, following the ideas in [28-31], as well as by considering $k$-order difference equations and $k$-order fractional difference equations with $k \geq 3$, see [32, 33].

\section{Data Availability}

The data used to support the theoretical findings of this study were obtained by means of the mini software package FIXPOINT and are available from the corresponding author upon request.

\section{Conflicts of Interest}

The author declares that he has no conflicts of interest.

\section{Acknowledgments}

This research was supported by the Department of Mathematics and Computer Science, Technical University of Cluj-Napoca, North University Centre at Baia Mare, through the Research Grant no. 2/2020.

\section{References}

[1] R. DeVault, G. Ladas, and S. W. Schultz, "On the recursive sequence $x_{n+1}=\left(A / x_{n}\right)+\left(1 / x_{n-2}\right)$," Proceedings of the American Mathematical Society, vol. 126, no. 11, pp. 32573261, 1998.

[2] R. M. Abu-Saris and R. DeVault, "Global stability of $y_{n+1}=A+\left(y_{n} / y_{n-k}\right)$, , Applied Mathematics Letters, vol. 16, no. 2, pp. 173-178, 2003.

[3] H. M. El-Owaidy, A. M. Ahmed, and M. S. Mousa, "On asymptotic behaviour of the difference equation $x_{n+1}=\alpha+\left(x_{n-k} / x_{n}\right)$," Applied Mathematics and Computation, vol. 147, no. 1, pp. 163-167, 2004.

[4] S. Stević, "On the recursive sequence $x_{n+1}=\alpha+\left(x_{n-1}^{p} / x_{n}^{p}\right)$," Journal of Applied Mathematics and Computing, vol. 18, no. 12, pp. 229-234, 2005.

[5] S. Stević, "On the recursive sequence $x_{n+1}=A+\left(x_{n}^{p} / x_{n-1}^{p}\right)$," Discrete Dynamics in Nature and Society, vol. 2007, p. 9, Article ID 34517, 2007. 
[6] S. Stević, "On the recursive sequence $x_{n+1}=A+\left(x_{n}^{p} / x_{n-1}^{r}\right)$," Discrete Dynamics in Nature and Society, vol. 2007, p. 9, Article ID 40963, 2007.

[7] S. Stević, "On the recursive sequence $x_{n+1}=\max \{c$, $\left.\left(x_{n}^{p} / x_{n-1}^{p}\right)\right\}$," Applied Mathematics Letters, vol. 21, no. 8, pp. 791-796, 2008.

[8] M. Aloqeili, "On the difference equation $x_{n+1}=\alpha+$ $\left(x_{n}^{p} / x_{n-1}^{p}\right)$," Journal of Applied Mathematics and Computing, vol. 25, no. 1-2, pp. 375-382, 2007.

[9] I. A. Rus, "An iterative method for the solution of the equation $x=f(x, \ldots, x)$," Mathematica-Revue d'Analyse Numérique et de Théorie de l'Approximation, vol. 10, no. 1, pp. 95-100, 1981.

[10] M. Saleh and M. Aloqeili, "On the rational difference equation," Applied Mathematics and Computation, vol. 171, no. 2, pp. 862-869, 2005.

[11] M. Saleh and M. Aloqeili, "On the rational difference equation," Applied Mathematics and Computation, vol. 177, no. 1, pp. 189-193, 2006.

[12] A. M. Amleh, E. A. Grove, G. Ladas, and D. A. Georgiou, "On the recursive sequence $x_{n+1}=\alpha+\left(x_{n-1} / x_{n}\right)$," Journal of Mathematical Analysis and Applications, vol. 233, no. 2, pp. 790-798, 1999.

[13] E. Camouzis, R. DeVault, and G. Ladas, "On the recursive sequence $x_{n+1}=-1+\left(x_{n-1} / x_{n}\right)$," Journal of Difference Equations and Applications, vol. 7, no. 3, pp. 477-482, 2001.

[14] L. B. Cirić and S. B. Prešić, "On Prešić type generalization of the Banach contraction mapping principle," Acta Mathematica Universitatis Comenianae. New Series, vol. 76, no. 2, pp. 143-147, 2007.

[15] Y.-Z. Chen, "A Prešić type contractive condition and its applications," Nonlinear Analysis Theory, Methods \& Applications, vol. 71, no. 12, pp. e2012-e2017, 2009.

[16] E. M. Elabbasy, H. El-Metwally, and E. M. Elsayed, "On the difference equation $x_{n+1}=a x_{n}-b x_{n} /\left(c x_{n}-d x_{n-1}\right), " A d$ vances in Difference Equations, vol. 2006, p. 10, Article ID 82579, 2006.

[17] H. M. El-Owaidy, A. M. Ahmed, and M. S. Mousa, "On asymptotic behaviour of the difference equation $\left.x_{n+1}=\alpha+\left(x_{n-1}^{p} / x_{n}^{p}\right)\right)^{\prime \prime}$ Journal of Applied Mathematics and Computing, vol. 12, no. 1-2, pp. 31-37, 2003.

[18] V. L. Kocić and G. Ladas, "Global behavior of nonlinear difference equations of higher order with applications," in Mathematics and its ApplicationsKluwer Academic Publishers Group, Dordrecht, Netherlands, 1993.

[19] M. Păcurar, "Approximating common fixed points of PrešićKannan type operators by a multi-step iterative method," Analele Ştiinţifice ale Universităţii "Ovidius" Constanţa. Seria Matematică, vol. 17, no. 1, pp. 153-168, 2009.

[20] M. Păcurar, Iterative Methods for Fixed Point Approximation, Risoprint, Cluj-Napoca, Romania, 2010.

[21] M. Păcurar, "A multi-step iterative method for approximating fixed points of Prešić-Kannan operators," Acta Mathematica Universitatis Comenianae. New Series, vol. 79, no. 1, pp. 7788, 2010.

[22] M. Păcurar, "A multi-step iterative method for approximating common fixed points of Prešić-Rus type operators on metric spaces," Studia. Universitatis Babeş-Bolyai Mathematica, vol. 55, no. 1, pp. 149-162, 2010.

[23] M. Păcurar, "Fixed points of almost Prešić operators by a k-step iterative method," Analele ştiinţifice ale Universităţii "Al. I. Cuza" din Iaşi. Serie Nouă. Matematică, vol. 57, pp. 199-210, 2011.
[24] S. B. Prešić, "Sur une classe d' inéquations aux différences finites et sur la convergence de certaines suites," Publications de l'Institut Mathematique, vol. 5, no. 19, pp. 75-78, 1965.

[25] V. Berinde, H. Fukhar-ud-din, and M. Păcurar, "On the global stability of some $k$-order difference equations," Results in Nonlinear Analysis, vol. 1, no. 1, pp. 13-18, 2018.

[26] V. Berinde, Exploring, Investigating and Discovering in Mathematics, Birkhäuser, Basel, Switzerland, 2004.

[27] V. Berinde, "Iterative approximation of fixed points," Lecture Notes in Mathematics, Springer, Berlin, Germany, 2nd edition, 1912.

[28] P. Agarwal and A. A. El-Sayed, "Non-standard finite difference and Chebyshev collocation methods for solving fractional diffusion equation," Physica A: Statistical Mechanics and Its Applications, vol. 500, pp. 40-49, 2018.

[29] A. A. El-Sayed and P. Agarwal, "Numerical solution of multiterm variable-order fractional differential equations via shifted Legendre polynomials," Mathematical Methods in the Applied Sciences, vol. 42, no. 11, pp. 3978-3991, 2019.

[30] G.-C. Wu and D. Baleanu, "Stability analysis of impulsive fractional difference equations," Fractional Calculus And Applied Analysis, vol. 21, no. 2, pp. 354-375, 2018.

[31] D. Baleanu, G. C. Wu, Y. R. Bai, and F. L. Chen, "Stability analysis of Caputo-like discrete fractional systems," Communications in Nonlinear Science and Numerical Simulation, vol. 48, pp. 520-530, 2017.

[32] V. Berinde and M. Păcurar, "Stability of $k$-step fixed point iterative methods for some Prešić type contractive mappings," Journal of Inequalities and Applications, vol. 2014, Article ID 149, 2014.

[33] V. Berinde and M. Păcurar, "Two elementary applications of some Prešić type fixed point theorems," Creative Mathematics and Informatics, vol. 20, no. 1, pp. 32-42, 2011. 